\title{
A Composite Laminate Veneer Technique for Diastema Closure: A Report of Ten Cases
}

\author{
Emrullah BAHŞí ${ }^{1}$, Michele Callea ${ }^{2}$, Bayram İNCE $^{1}$, Marco Montanari ${ }^{3}$, Mehmet DALLI ${ }^{1}$, \\ Filippo Batteli ${ }^{3}$, Mehmet AKDOĞAN ${ }^{1}$, Clarich Gabriella ${ }^{2}$, İsmet Rezani TOPTANCI ${ }^{4}$, Piana \\ Gabriela ${ }^{3}$, İzzet YAVUZ ${ }^{4}$ \\ ${ }^{1}$ Dicle University, Faculty of Dentistry, Department of Restorative Dentistry, Diyarbakır, Turkey. \\ ${ }^{2}$ Maternal and Child Health Institute, Unit Maxillo Facial Surgery and Pediatric Dentisry, Trieste, Italy. \\ 3 University of Bologna, Unit of Dentistry for Disables Department of Oral Science, , Bologna, Italy. \\ ${ }^{4}$ Dicle University, Faculty of Dentistry, Department of Pediatric Dentistry, Diyarbakır, Turkey.
}

\section{Key Words}

Diastema closure, esthetic treatment, composite laminate.

\section{Correspondence:}

Emrullah Bahşi

Dicle University, Faculty of Dentistry

Restorative Dentistry

Diyarbakır, TURKEY.

e-mail: emrullahbahsi@hotmail.com

\section{Abstract}

Aim: Diastemas can be treated using periodontal, surgical, orthodontic, or prosthetic procedures. Composite laminate veneers can be applied to reduce or eliminate diastemas and represent a good aesthetic non-invasive alternative for these patients. This study presents the treatment results of 10 patients with diastemas between the anterior teeth of the maxilla and mandible with composite veneers.

Methodology: Ten patients with diastemas between the anterior maxillary and mandibular teeth complaining of esthetic problems were treated at the Department of Operative Dentistry, University of Dicle. The periodontal health of the patients was acceptable and no caries were detected. Patients were informed of the treatment choices and composite resin veneers were selected as the better approach for each case. The presence of a diastema causes esthetic problems, especially in adolescents. Prosthetic and conservative methods are usually used to treat diastemas because these approaches are minimally invasive. The direct laminate technique has the advantages of low cost, treatment reversibility, and easy repair of the restoration intraorally. The direct composite laminate technique has become more effective because of improvements in adhesive dentistry.

Conclusion: This study describes a direct composite laminate technique for the restoration of the anterior teeth of 10 patients with diastemas. These restorations were conservative and provide an inexpensive, one-visit aesthetic treatment for anterior teeth.

(Int Dent Res 2013;2(3):67-74)

\section{Introduction}

Anterior diastemas can have a negative effect on a person's smile, and many patients improve their appearance with either orthodontic treatment or restorative bonding of veneers, crowns, or composite resins. Increasing patient demand for optimal aesthetics has resulted in the extensive 
utilization of composite resin bonding in the anterior region because it involves less invasive procedures. To achieve a successful directly bonded restoration, the dentist must have broad knowledge of adhesive dentistry, which involves the manipulation of composite resins, proper preparation, and an understanding of the optical properties of the natural tooth and their relationship to anatomical morphology. Composite resin bonding to close a diastema is a conservative, relatively inexpensive technique to correct the smile and is often carried out in a single visit in many dental practices (1).

A diastema is a space between two teeth. Many mammal species have diastemas as a normal feature, most commonly between the incisors and molars (2). A diastema can result from differences in the dental dimensions and spaces in the dental arch. Dental loss and the passive eruption of teeth can cause diastemas that can be treated with periodontal, surgical, orthodontic, or prosthetic procedures (3). Fixed dental prostheses are preferred when treating patients with such anomalies, although the disadvantages of tooth preparation, the cost of the restoration, and time for fabrication sometimes lead the clinician to try another type of restoration. Composite laminate veneers are an alternative for these patients (4). This study presents the treatment of diastemas between anterior maxillary and mandibular teeth with composite veneers.

\section{Case Series}

Ten patients with diastemas between maxillary and mandibular teeth with aesthetic complaints were treated at the Department of Operative Dentistry, University of Dicle. A detailed dental and medical history was obtained from the patients. The periodontal health of the patients was acceptable, and no caries were detected. The patients were informed of the treatment choices for their diastemas and composite resin veneers were selected as the better approach for each case. All diastemas were treated following the same steps. The first step was periodontal treatment and then color shading was performed with a Vita Easy Shade spectrophotometer (Vita Zahnarzt, Almanya). The dimensions of the space and the teeth near the space were measured. The enamel surfaces of the teeth were etched with $35 \%$ orthophosphoric acid (Scotchbond; 3M ESPE, St. Paul, MN, USA) for $60 \mathrm{~s}$ and then rinsed with water. Primer was applied in two coats and left undisturbed for $20 \mathrm{~s}$; then, the solvent was evaporated for $10 \mathrm{~s}$. Bonding was applied twice, spread using a gentle stream of oilfree air for 2 s (Clearfil SE Bond; Kuraray, Osaka, Japan), and polymerized for $10 \mathrm{~s}$ using a light- emitting diode (LED) light-curing unit (Elipar Freelight, 3M ESPE, Germany). Then, the composite resin (Clearfil Majesty Esthetic; Kuraray, Osaka, Japan) was applied and polymerized for $20 \mathrm{~s}$. Finishing was performed using abrasive discs (SofLex, 3M ESPE, St. Paul, MN, USA). After finishing the first restoration, the second restoration was completed following the same steps. All treated patients were informed about proper oral hygiene and were followed every 6 months.

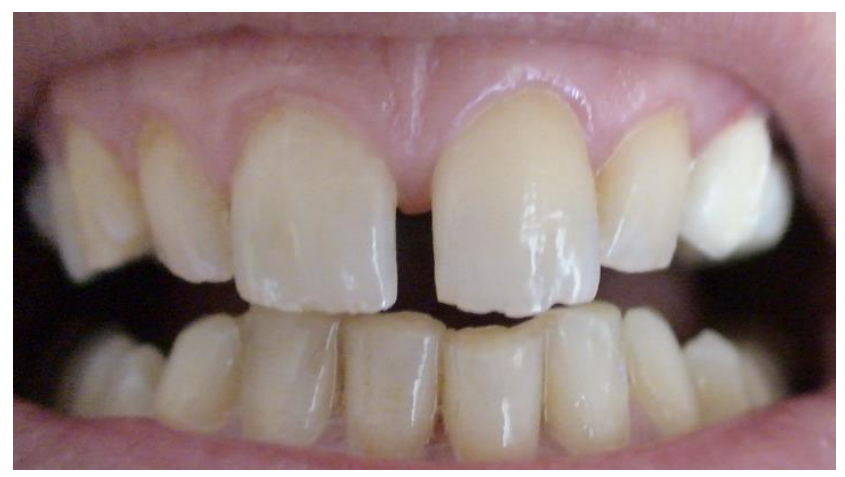

Figure 1a. The appearance of case 1 with diastemata between upper central incisors before treatment

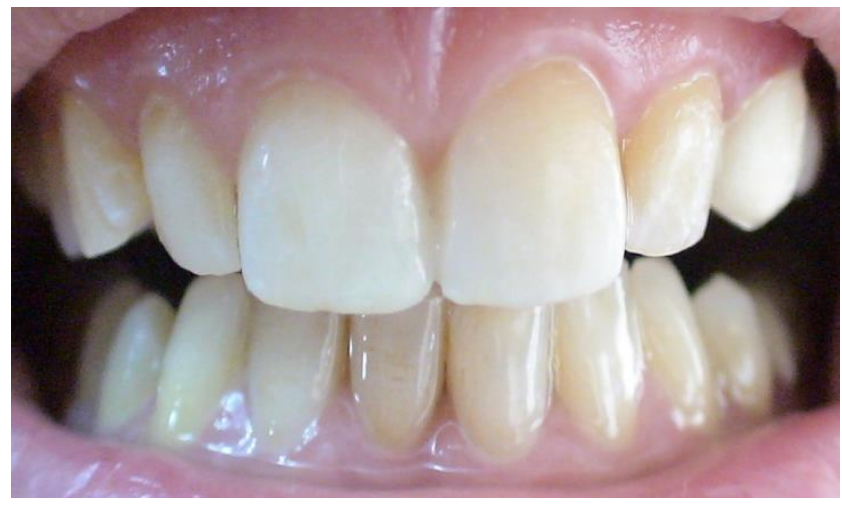

Figure 1b. The appearance of case 1with diastemata between upper central incisors after treatment

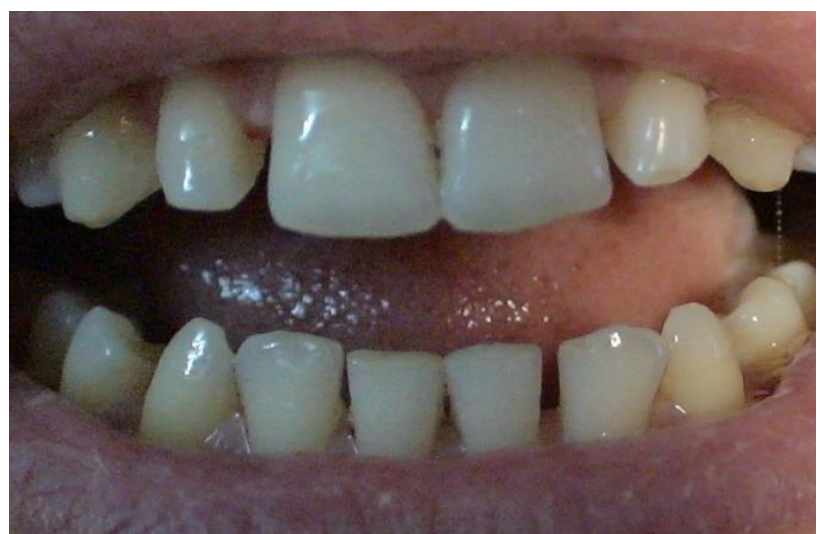

Figure 2a. The appearance of case 2 with diastemata between lower incisors before treatment 


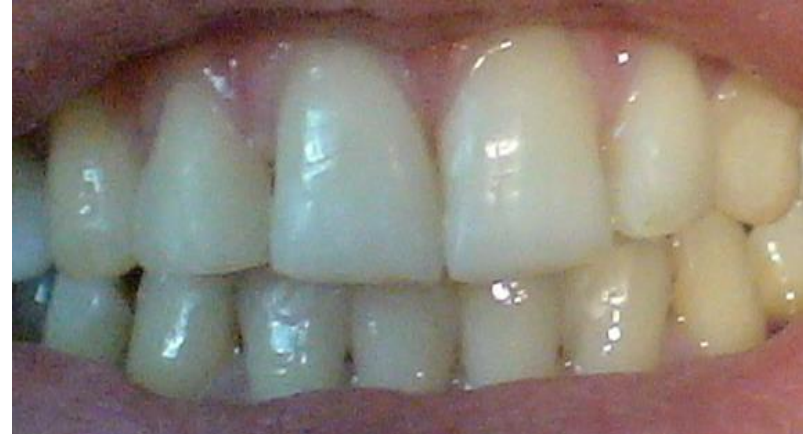

Figure 2b. The appearance of case 2 with diastemata between lower incisors after treatment

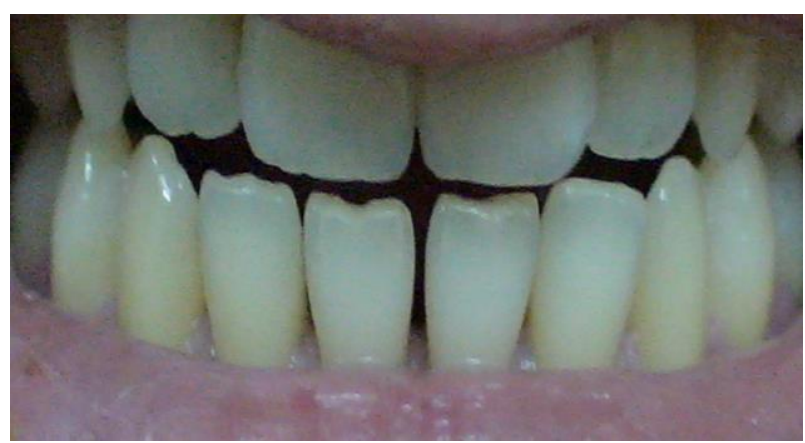

Figure 3a. The appearance of case 3 with diastemata between lower central incisors before treatment

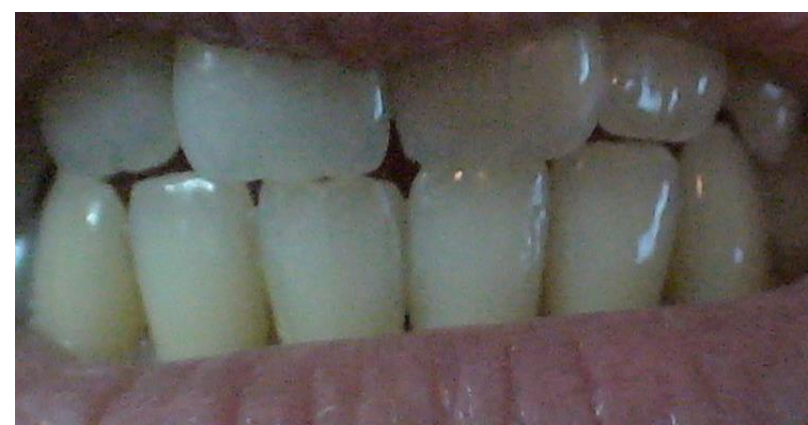

Figure $\mathbf{3 b}$. The appearance of case 3 with diastemata between lower central incisors after treatment

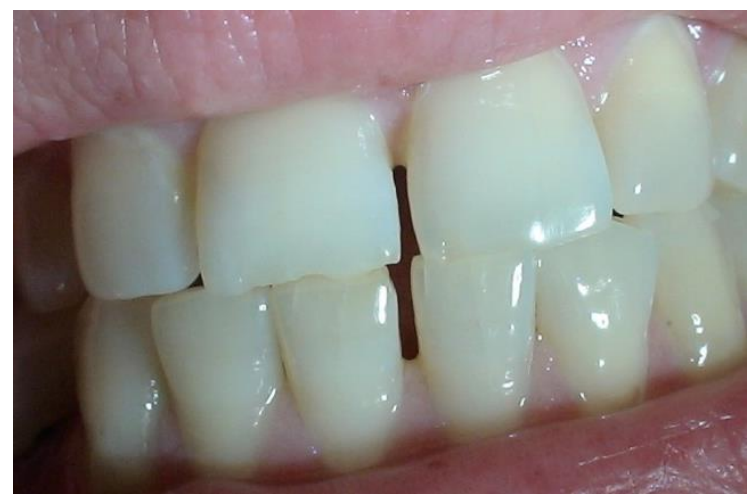

Figure 4a. The appearance of case 4 with diastemata between both upper and lower central incisors before treatment

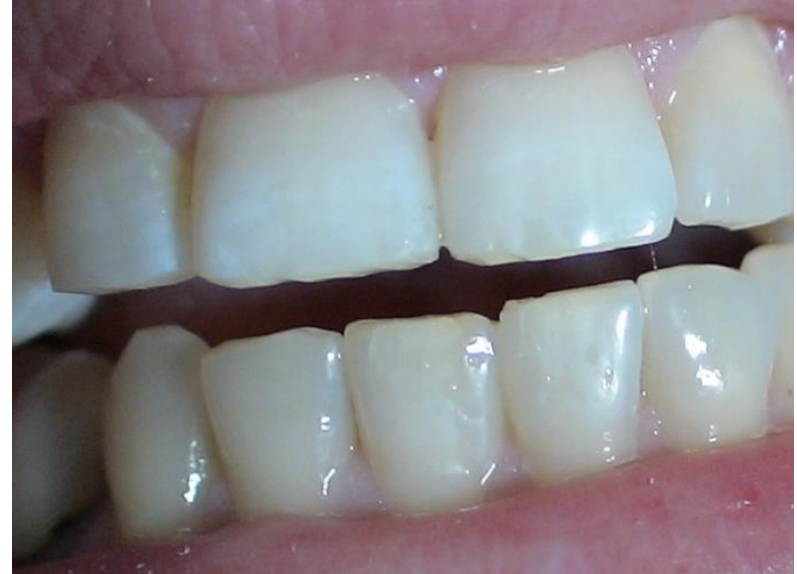

Figure 4b. The appearance of case 4 with diastemata between both upper and lower central incisors after treatment

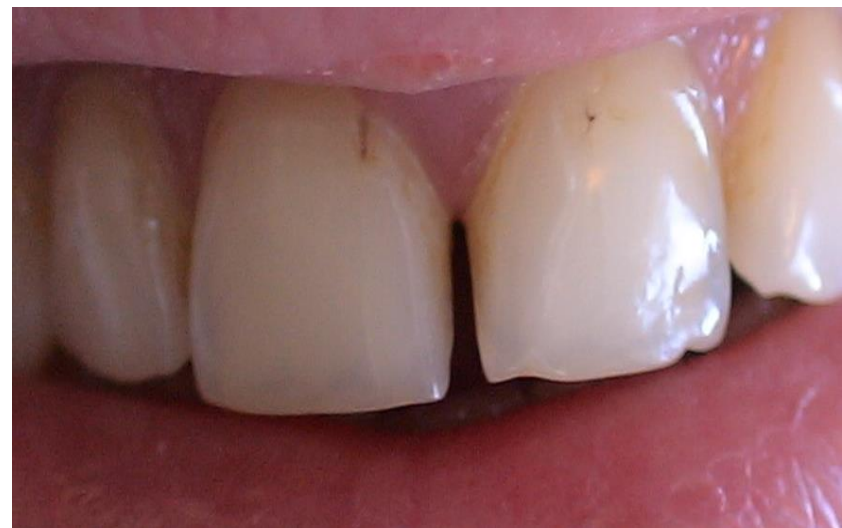

Figure 5a. The appearance of case 5 with diastemata between upper central incisors before treatment

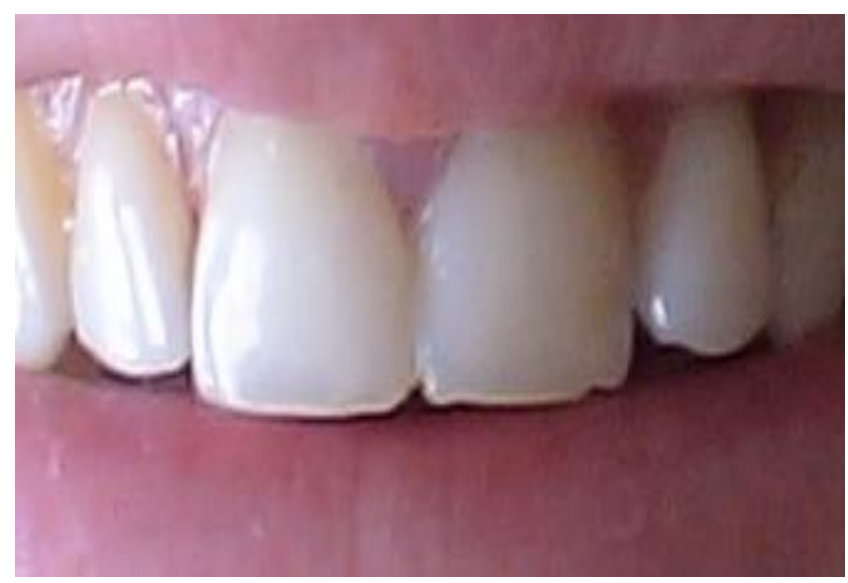

Figure 5b. The appearance of case 5 with diastemata between upper central incisors after treatment 


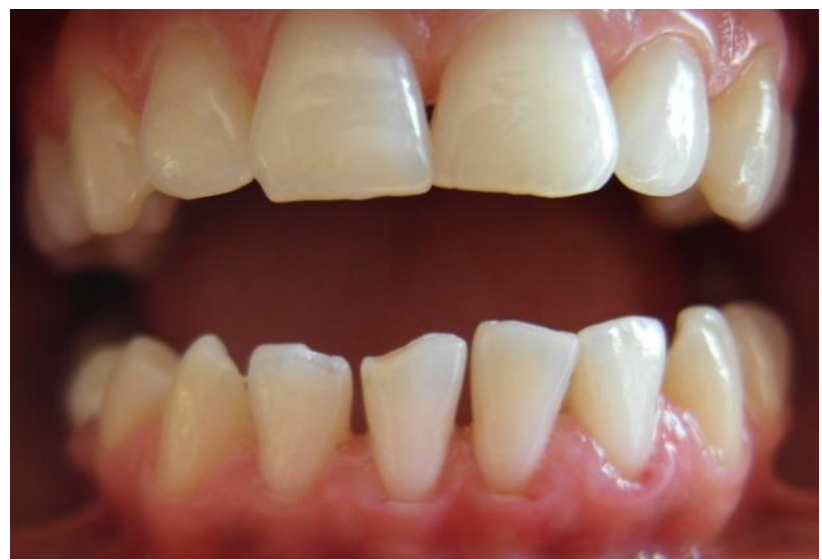

Figure 6a. The appearance of case 6 with diastemata between lower incisors before treatment

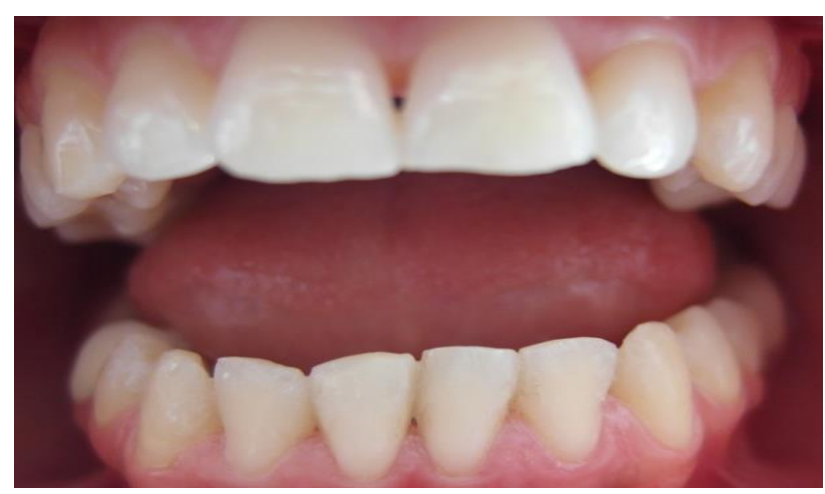

Figure $\mathbf{6 b}$. The appearance of case 6 with diastemata between lower incisors after treatment

\section{Discussion}

The effective control of dental plaque is an Recently, the physical and optical properties of composite resins have been developed to the point where they often represent a first-line therapeutic approach. The indications for the use of composite resin procedures have increased and include fractured, discolored teeth, diastema closure, correcting shape, and malpositioned teeth (5).

The presence of a diastema causes esthetic problems, especially in adolescents. Prosthetic and conservative methods are usually used to treat diastemas, especially because these approaches are minimally invasive. The direct laminate technique has the advantages of low cost, treatment reversibility, and easy repair of the restoration intraorally (6). In this study, composite laminates were preferred for restorations because of their low cost, good aesthetics, and repairability (Figs. 1-10).

Freehand direct resin composites provide an aesthetic, conservative approach for diastema closure and should be part of the armamentarium of every cosmetic dentist. By applying a step-by-step approach to diastema closure when using direct resin composites, the practitioner has the opportunity to incorporate shade selection, tooth preparation, material selection, composite layering, material blending, proper gingival contouring, and polishing to close diastemas in a predictable, efficient manner in daily practice (7).

Diastema closure with a direct composite resin challenges our artistic and technical abilities and calls on our knowledge of the smile design principles of proportion, symmetry, harmony, and tooth morphology $(8,9)$. Diastema closure is a frequently sought technique-sensitive cosmetic procedure, which if performed correctly, enhancing dental-facial aesthetics, and can result in psychological and social benefits to our patients (10).

Although composite resins are esthetic and easy to manipulate, they have some undesirable properties, such as staining, microleakage, low abrasion resistance, and plaque accumulation, so they are more appropriate to use for anomalies limited to enamel and as provisional restorations (4). Increasing the particle size of the resin by decreasing the proportion of organic filler matrix can decrease the change in color (11). Hybrid composite resins were used in this study because they have good mechanical resistance and good esthetics $(12,13)$. The direct composite laminate technique has become more effective because of improvements in adhesive dentistry. Most manufacturers of dental adhesives offer both totaletch and self-etch adhesives. The strongest bonding to enamel is obtained with total-etch adhesives (14), while two-step self-etch adhesives perform better on deep and superficial dentine in the presence of humidity. In our patients, the total-etch approach was followed using a self-etch adhesive to obtain better bonding to enamel (15).

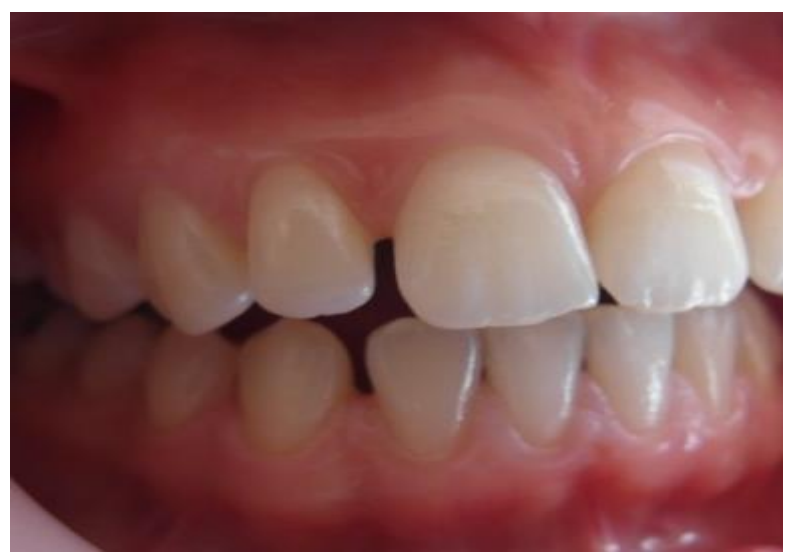

Figure 7a. The right side appearance of case 7 with diastemata between upper and lower teeth before treatment. 


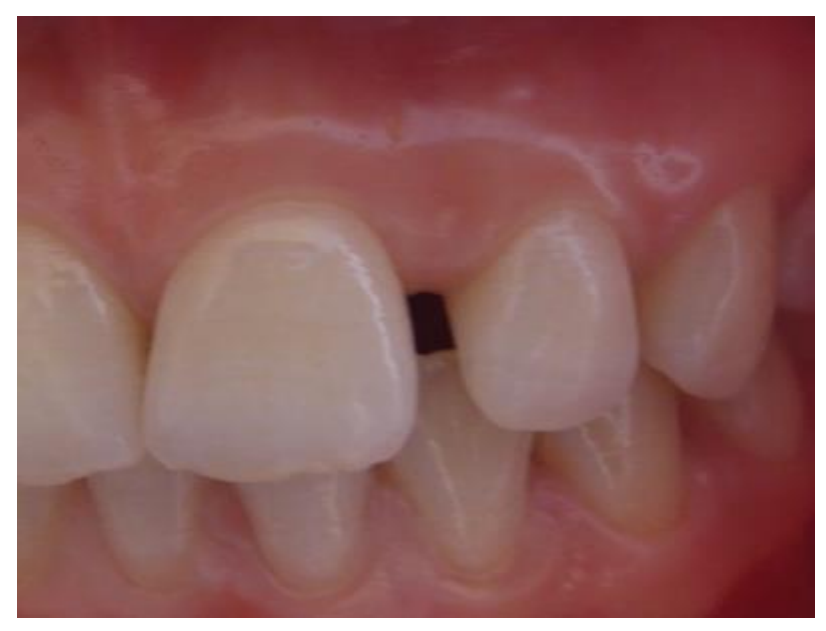

Figure 7b. The left side appearance of case 7 with diastemata between upper and lower incisors before treatment.

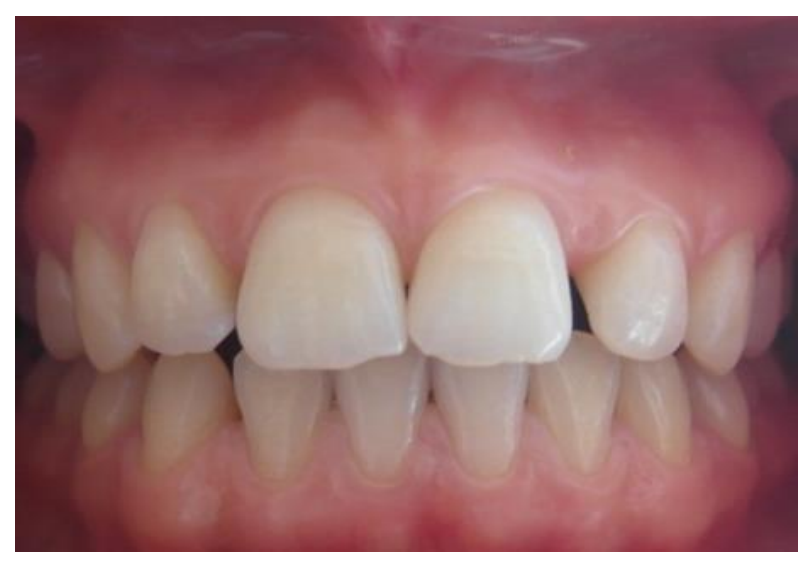

Figure 7c. The front side appearance of case 7 with diastemata between upper and lower teeth before treatment

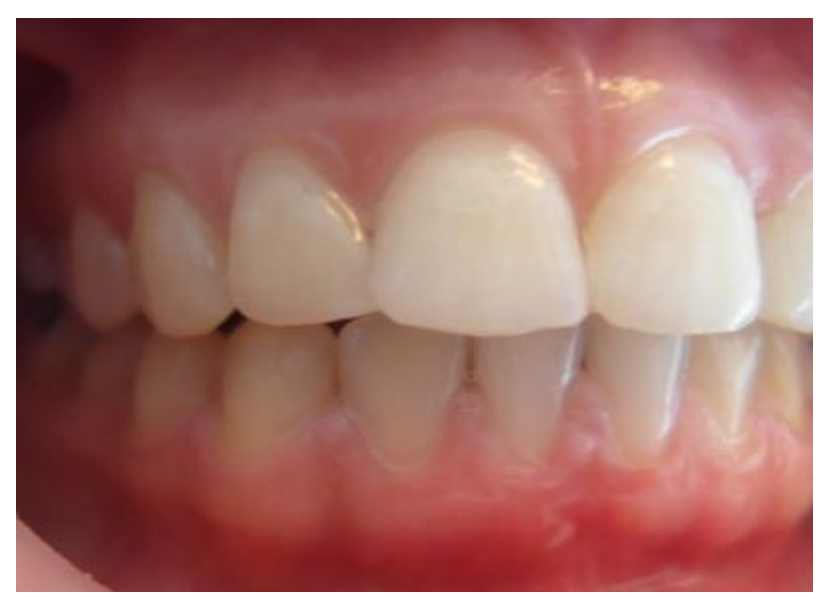

Figure 7d. The right side appearance of case 7 with diastemata between upper and lower teeth after treatment

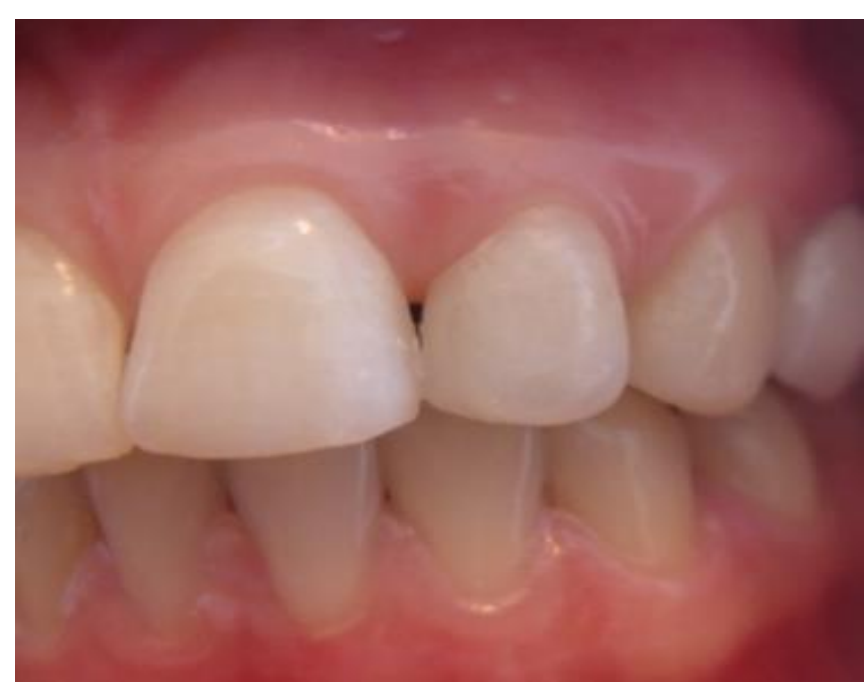

Figure 7e. The right side appearance of case 7 with diastemata between upper and lower incisors after treatment

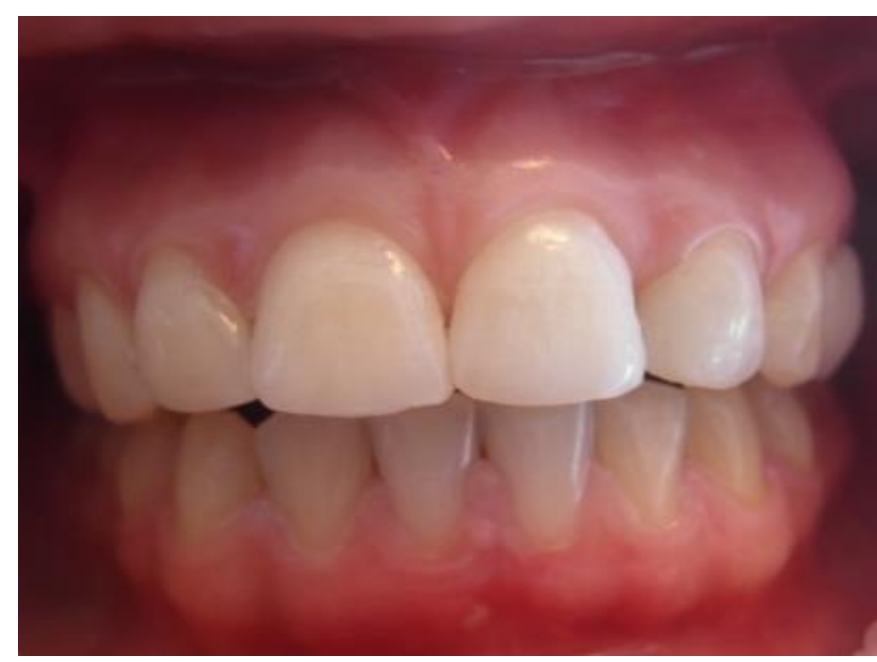

Figure 7f. The front side appearance of case 7 with diastemata between upper and lower teeth after treatment

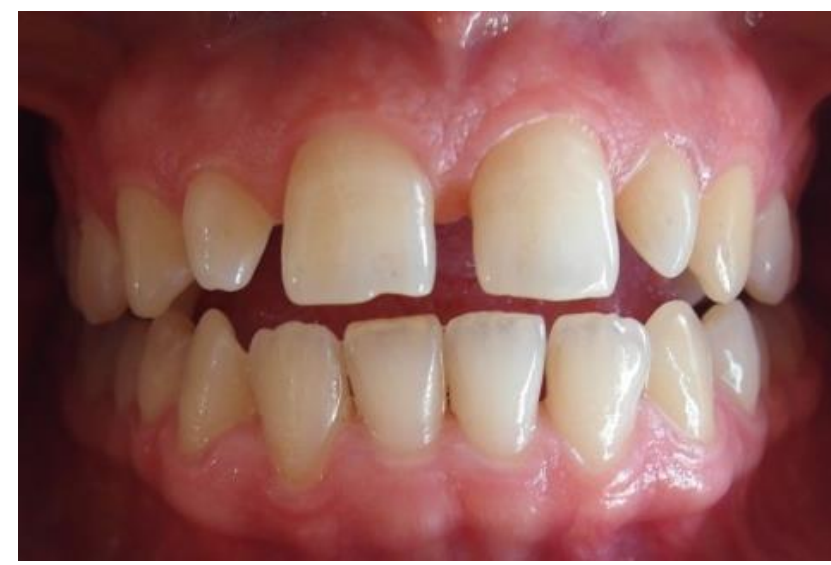

Figure 8a. The appearance of case 8 with diastemata between upper teeth before treatment 


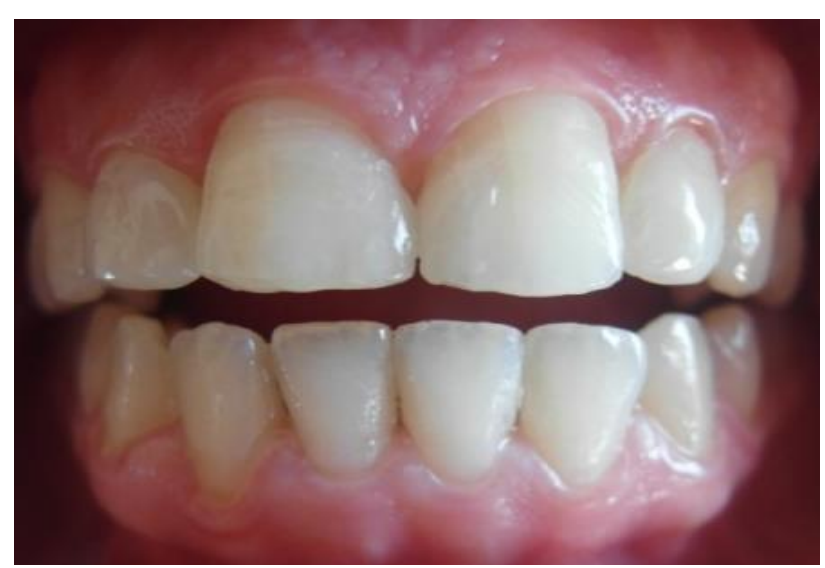

Figure $\mathbf{8 b}$. The appearance of case 8 with diastemata between upper teeth after treatment.

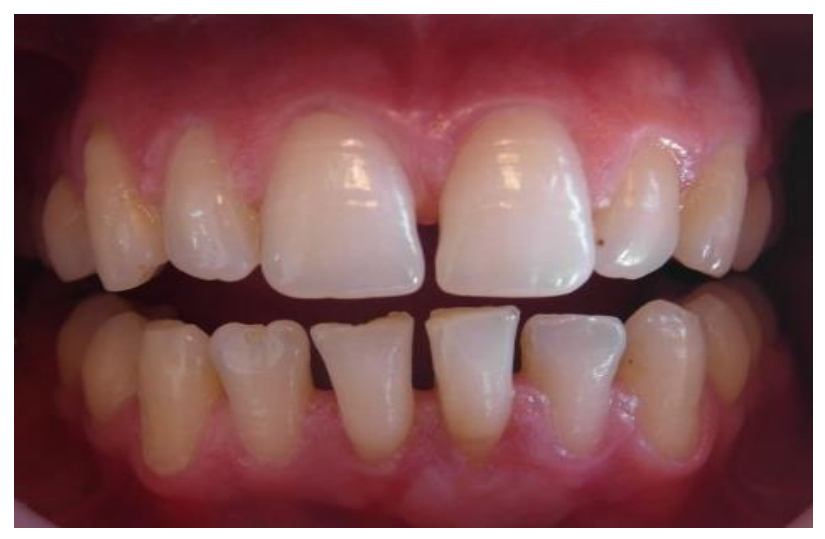

Figure 9a. The appearance of case 9 with diastemata between upper and lower teeth before treatment

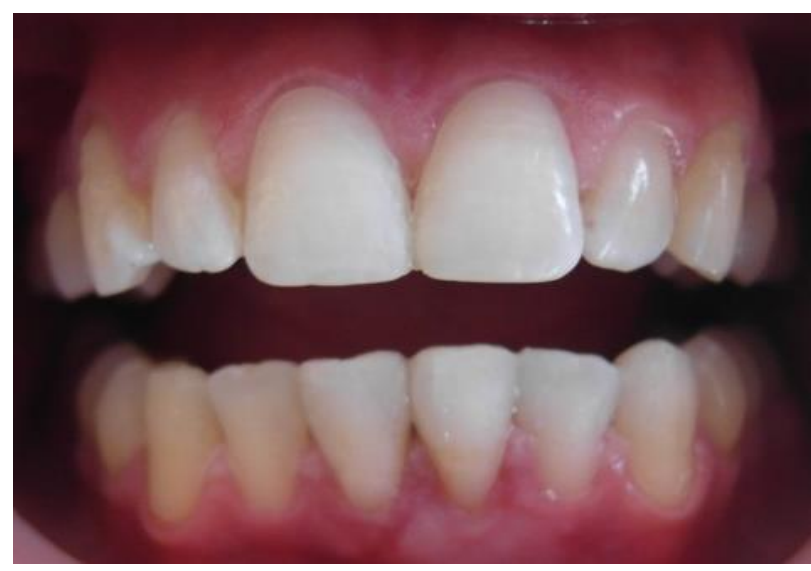

Figure $\mathbf{9 b}$. The appearance of case 9 with diastemata between upper and lower teeth after treatment

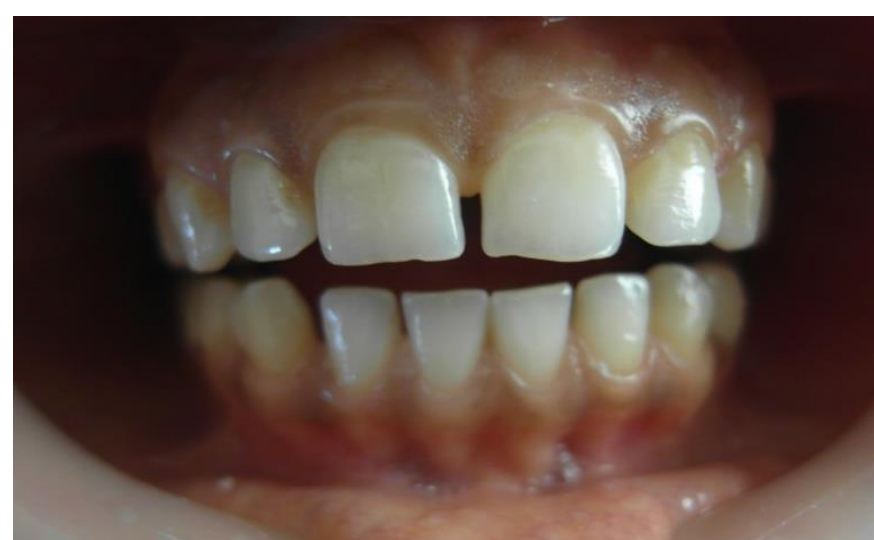

Figure 10a. The right front side appearance of case 10 with diastemata between upper and lower teeth before treatment

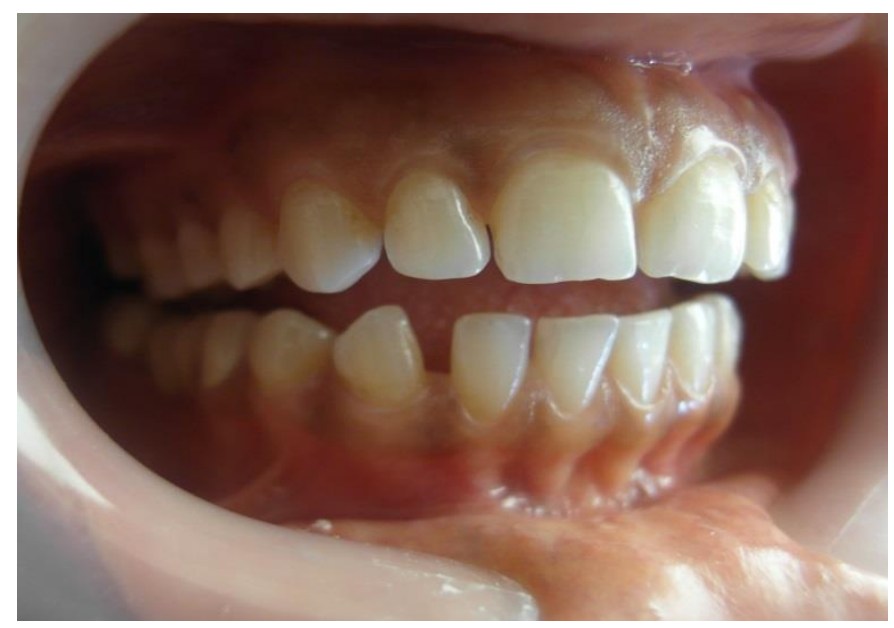

Figure 10b. The right side appearance of case 10 with diastemata between upper and lower teeth before treatment

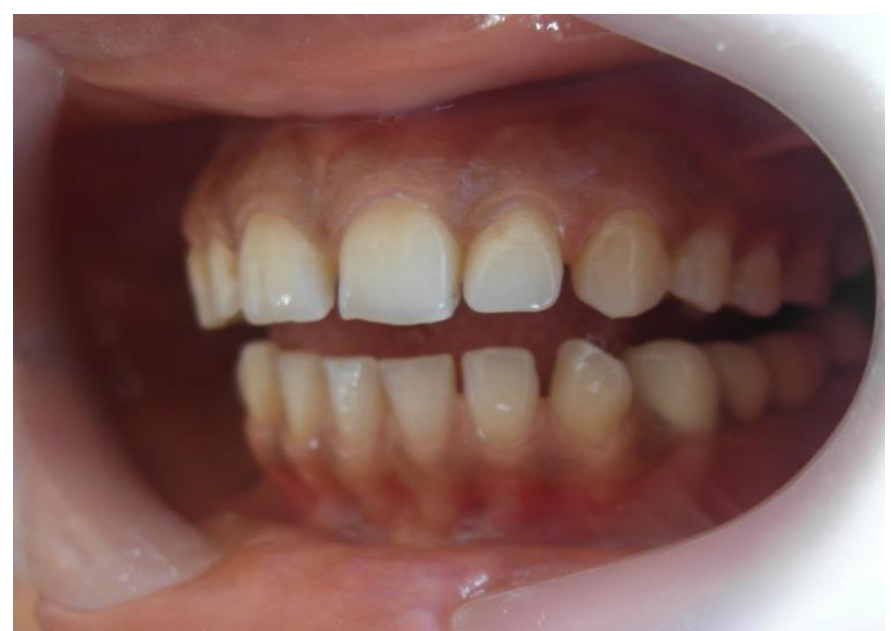

Figure 10c. The left side appearance of case 10 with diastemata between upper and lower teeth before treatment 


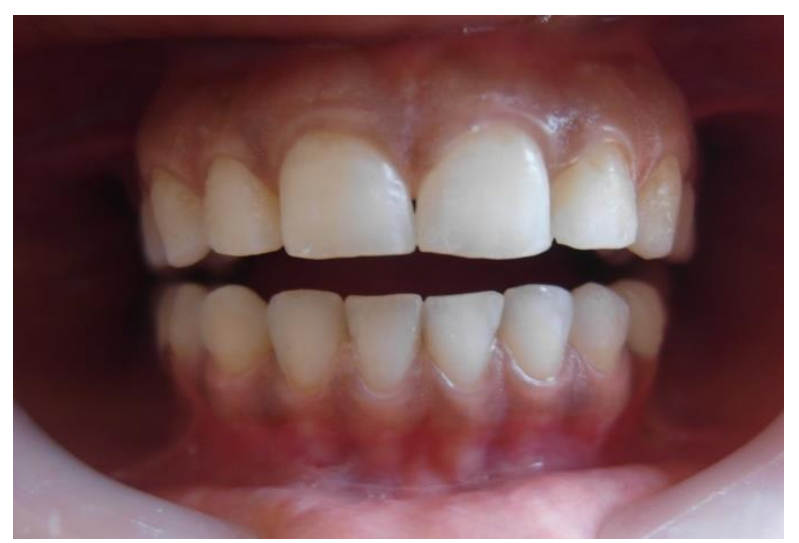

Figure 10d. The front side appearance of case 10 with diastemata between upper and lower teeth after treatment.

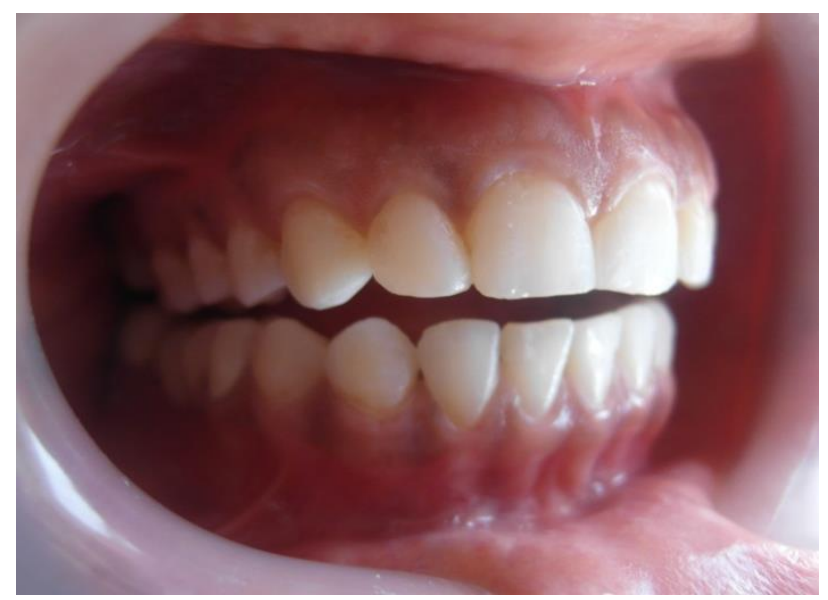

Figure 10e. The right side appearance of case 10 with diastemata between upper and lower teeth after treatment

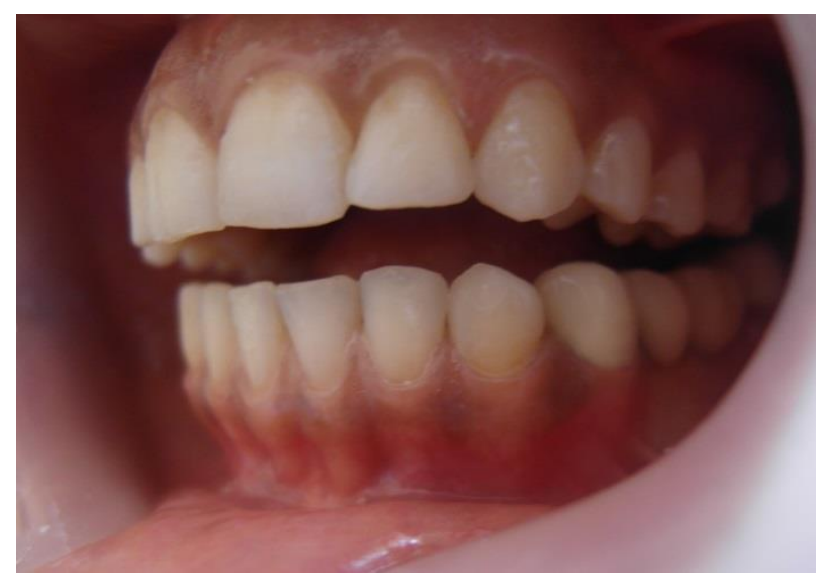

Figure 10f. The left side appearance of case 10 with diastemata between upper and lower teeth after treatment.
If composite resins are placed incorrectly, difficulty flossing, increased plaque retention, and gingival irritation can affect the patient's periodontal health and the tooth-restorative margin can develop microleakage and caries $(16,17)$. Increasingly, the medical and dental literature mentions a possible relationship between periodontal and systemic health $(18,19)$. Care must be taken to ensure that we decrease the potential of gingival irritation and inflammation in our restorative procedures (20).

Composite resins have very good physical features, marginal integrity, and aesthetics $(21,22)$. Compared with ceramic restorations, resin composites do not cause abrasion of antagonist teeth and do not have an unrepairable fracture potential (21-23). Comparing this method with the indirect method, the advantage of this method is that it allows different treatment methods in the future. Compared with other restorative materials, the important advantages of composite resins are their aesthetics, no risk of mechanical performance change, and possibility of intraoral repair (21).

\section{Conclusion}

This clinical report describes a direct composite laminate technique for restoring the anterior teeth of ten patients with diastemas. These restorations are conservative and enable a one-visit aesthetic treatment alternative for anterior teeth. A direct composite laminate technique might be an important treatment choice compared with fixed dental prostheses.

\section{Acknowledgments}

The authors deny any conflicts of interest related to this study.

\section{References}

1. Ho CCK. Diastema closure with a micro-hybrid composite resin. Dental Practice 2006;156-160.

2. http://dentistry.about.com/od/glossary/g/diastem a.htm.

3. de Araujo EM Jr, Baratieri LN, Monteiro S Jr, Vieira LC, de Andrada MA. Direct adhesive restoration of anterior teeth: Part 2. Clinical protocol. Prac Proced Aesthet Dent 2003;15:3517.

4. Yüzügüllü $B$, Tezcan S. Comparative indications of laminate veneer restoration types used for 
colored and eroded teeth. Cumhuriyet University Faculty of Dentistry 2005;8(2):133-137.

5. Fahl N. A polychromatic composite layering approach for solving a complex class iv/direct veneer-diastema combination: Part 1. Pract Proced Aesthet Dent 2006;18(10):A-G

6. Magne $P$, Besler UC. Porcelain versus composite inlays/onlays effects of mechanical loads on stress distribution adhesion and crown flexure. Int J Periodontics Restor Dent 2003;23:543-555.

7. Vargas, M. A step-by-step approach to a diastema closure: A dual-purpose technique that manages black triangles. J Cosmetic Dent 2010;26(3):40-45.

8. Vanini L. Light and color in anterior composite restorations. Pract Periodontics Aesthet Dent 1996;8:673-682.

9. Peyton $\mathrm{JH}$. Finishing and polishing techniques: direct composite resin restorations. Pract Proced Aesthet Dent 2004;16:293-298.

10. Jones L, Robinson M. Case study: esthetic and biologic management of diastema closures using porcelain bonded restorations for excellent and predictable results. AACD Journal Of Cosmetic Dentistry 2002;18(3):73-84.

11. Sarac D, Sarac YS, Kulunk S, Ural C, Kulunk T. The effect of polishing techniques on the surface roughness and color change of composite resins. J Prosthet Dent 2006;96:33-0.

12. Garber DA, Goldstein RE, Feinman RA. Porcelain Laminate Veneers. Quintessence Publishing, Chicago; 1988.

13. Jordan RE. Mosby-Year Book. 2. St. Louis: 1993. Esthetic Composite Bonding Techniques and Materials.

14. Perdigao J, Gomes G, Duarte S, Lopes MM. Enamel bond strengths of pairs of adhesives from the same manufacturer. Oper Dent 2005;30:4929.

15. Sauro S, Pashley DH, Montanari M, Chersoni S, Carvalho RM, Toledano M, Osorio R, Tay FR, Prati C. Effect of simulated pulpal pressure on dentin permeability and adhesion of self-etch adhesives. Dental Materials 2007;23(6):705-713.

16. for predictable restoration of anterior teeth with composite resin. Pract Periodont Aesthet Dent 1995;7(8):13-21.

17. Clark D. Correction of the "black triangle" restoratively driven papilla regeneration. Dentistry Today 2009;150-155.

18. Badder $\mathrm{H}$. Inflammatory suppression: rationale and implications for the general practice. Dentistry Today Nov. 2008.

19. Beck J, Garcia R, Heiss G, et al. Periodontal disease and cardiovascular health. J Periodontol 1996:67(suppl 10):1123-1137.

20. Pollak M. Aesthetic diastema closure utilizing a novel matrix system. Oral Health group 2010-0301.
21. inlays/onlays: effects of mechanical loads on stress distribution, adhesion and crown flexure. Int J Periodontics Restorative Dent 2003;23:54355.

22. Nakamura $T$, Imanishi $A$, Kashima $H$, Ohyama $T$, Ishigaki S. Stress analysis of metal-free polymer crowns using the three-dimensional finite element method. Int J Prosthodont 2001;14:4015.

23. Ku CW, Park SW, Yang HS. Comparison of the fracture strengths of metal- ceramic crowns and three ceromer crowns. J Prosthet Dent 2002;88:170-175. 